\title{
Radiation-dose knowledge needs improving
}

$\mathrm{T}$ oday's doctors and patients are well aware that radiation exposure accompanies essential procedures such as X-rays and CT scans, but recent surveys suggest that this awareness does not always translate into precise knowledge of dosage limits among Canadian clinicians.

The latest indication of this gap was evident in a survey of 92 staff in The Ottawa Hospital's diagnostic imaging department about their knowledge of radiation dose and risk in common exam procedures. Only $48 \%$ of respondents got more than half the questions correct. Only 23\% knew the dose of both single-view and two-view chest $\mathrm{X}$-rays, $50 \%-70 \%$ underestimated the dose from common studies and $50 \%$ $75 \%$ underestimated the risk of fatal cancer, according to the survey results in the November 2014 issue of Insights into Imaging.

Dr. William Miller, vice-president of the Canadian Association of Radiologists (CAR), who was among those who answered the survey, interpreted the results as a pointed reminder of just how much there is to know about radiation dosage, a matter that remains front-and-centre within CAR.

"It certainly has been a topic in the literature, at meetings, and in every venue where a radiologist might participate" says Miller, who is chair of Diagnostic Imaging at The Ottawa Hospital. "It's been a push from us to our membership to get them to take radiation safety seriously."

The findings in the Ottawa study echo a comparable survey of 127 patients, 32 referring physicians, and 30 medical students conducted by a group of Ontario imaging specialists and published in 2012 by the Canadian Association of Radiologists Journal. In this survey, more than a quarter of the responding physicians were unaware that mammography employs ionizing radiation, while some $92 \%$ of patients reported that they were not told of any risks associated with radiation procedures.

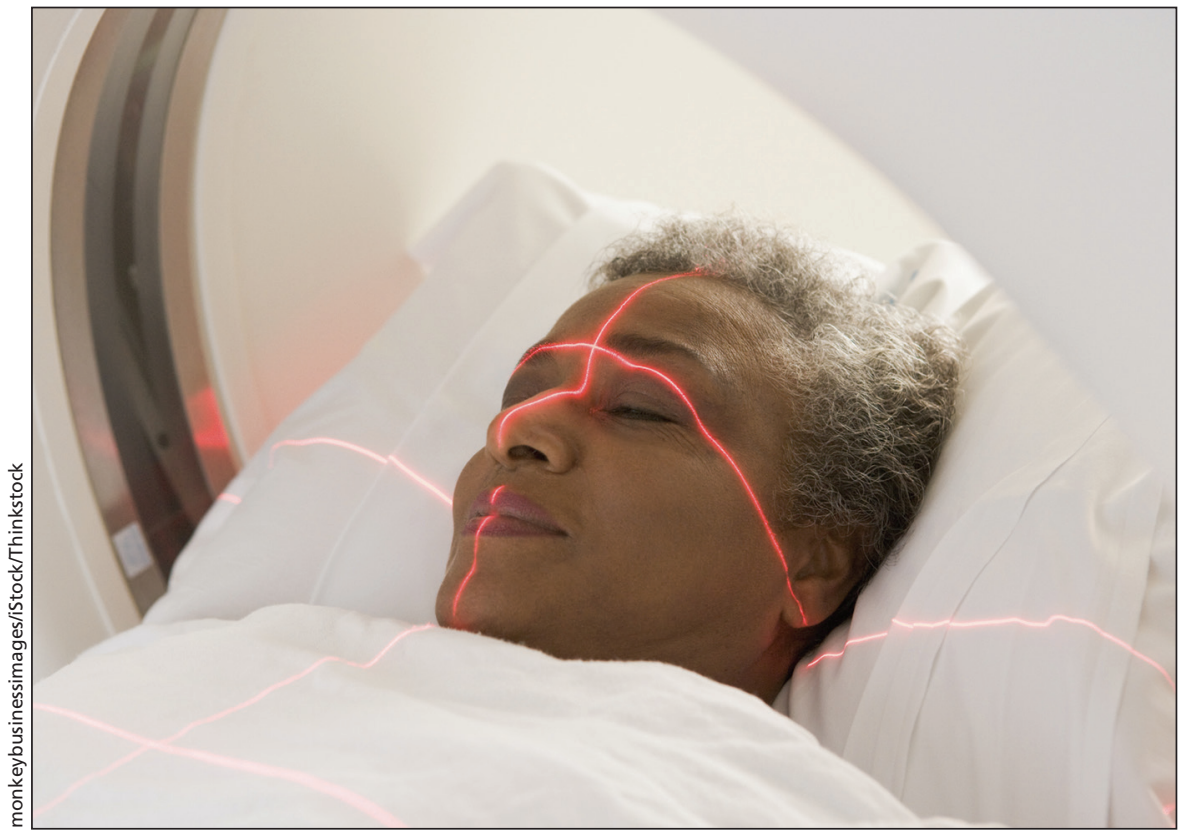

The Canadian Association of Radiologists is urging its members to "take radiation safety seriously."

The Ottawa survey's co-investigator Dr. John Ryan says it was an attempt to sample what is understood by people who regularly deal with radiation. "This was done to assess the knowledge that people have - not just the awareness - as well as the ability to communicate to a patient." Patients confronting tests with radiation now pointedly ask for alternatives that could lower or eliminate this dosage, he adds.

It is increasingly possible to honour such requests, says Dr. Blair Macdonald, a staff radiologist with The Ottawa Hospital. "CT usage in North America has plateaued since 2007, after 20 years of constant growth, and the doses per exam have dropped significantly," he says. "In all cases we are using less than $50 \%$ of the radiation per exam and in some cases less than $10 \%$ of the radiation per exam - compared with seven years ago."

Device manufacturers like GE Healthcare and Philips take some of the credit. GE touts Toronto's Hospital for Sick Children as an institution that minimizes the radiation exposure of its young clientele by capturing CT images in "slices" as opposed to a continuous body scan. The result dramatically lowers the dose with little or no compromise in image quality.

Such progress must be conveyed to the medical community, says Dr. Benjamin Chow, director of Cardiac Imaging at the University of Ottawa Heart Institute. He has led by example through innovations like computer software that extracts high resolution imagery from scans made with ever smaller amounts of energy.

"There's technology coming down the pipeline whereby risks of imaging will be further reduced," he says. Everyone with a vested interest in these techniques should be aware of advances that reduce patient risk, he says. And physicians performing the tests should be well informed of that risk.

"When I pick one test over another," he says, "I have to weigh the risks of the procedure and the diagnostic potential." — Tim Lougheed, Ottawa, Ont. 